\title{
Research Article \\ Optimal Control Systems by Time-Dependent Coefficients Using CAS Wavelets
}

\author{
Taher Abualrub, Ibrahim Sadek, and Marwan Abukhaled \\ Department of Mathematics, American University of Sharjah, Sharjah, UAE \\ Correspondence should be addressed to Taher Abualrub, abualrub@aus.edu
}

Received 7 July 2009; Accepted 23 November 2009

Recommended by M. A. Petersen

\begin{abstract}
This paper considers the problem of controlling the solution of an initial boundary-value problem for a wave equation with time-dependent sound speed. The control problem is to determine the optimal sound speed function which damps the vibration of the system by minimizing a given energy performance measure. The minimization of the energy performance measure over sound speed is subjected to the equation of motion of the system with imposed initial and boundary conditions. Using the modal space technique, the optimal control of distributed parameter systems is simplified into the optimal control of bilinear time-invariant lumped-parameter systems. A wavelet-based method for evaluating the modal optimal control and trajectory of the bilinear system is proposed. The method employs finite CAS wavelets to approximate modal control and state variables. Numerical examples are presented to demonstrate the effectiveness of the method in reducing the energy of the system.
\end{abstract}

Copyright (C) 2009 Taher Abualrub et al. This is an open access article distributed under the Creative Commons Attribution License, which permits unrestricted use, distribution, and reproduction in any medium, provided the original work is properly cited.

\section{Introduction}

Dynamic stability related to parametric resonance is a very important factor in structural dynamics. For example, instability caused by parametric resonance is believed to be the reason for the famous Tacoma bridge collapse in 1940 [1]. A suitable control of the coefficients may provide an effective protection against this phenomenon.

Control in the coefficients is known to be a very effective method in structures governed by elliptic equations [1]. However, not much information is known about the effect produced by control in coefficient for hyperbolic equations $[2,3]$. In this paper, we study a control problem for a structure dynamic system governed by a hyperbolic equation where the control is a time dependent coefficient.

The model considered in this work is motivated by recent developments in the area of smart materials [4]. The properties of these materials can be changed by applying external fields, such as electrical, magnetic, or temperature; this is referred to as a phase transformation. 
A structure made with such a material is considered where control consists of eliminating a transient disturbance in the structure by varying the material properties in the response to the deformation. The modal dynamic of a structure is governed by a scalar wave equation, where the control variable is the sound speed in the medium. The basic bilinear optimal control problem becomes the minimization of the energy function of the system in a given period of time with a minimum sound speed. Using modal expansion, the optimal control of the distributed parameter system is reduced to the optimal control of a bilinear time-varying lumped parameter system. The parameterization approach is used to approximate the state-variable and each component of the control variable using finiteterm wavelets with unknown coefficients. Therefore, the quadratic problem is transformed into a mathematical programming problem with the objective of minimizing the unknown coefficients to give suboptimal solution of the problem. A necessary condition for the optimality of the unknown coefficients is derived as a system of linear algebraic equations for which the solution is used to obtain the optimal control sound speed and optimal state function.

The bilinear system is a kind of nonlinear system where some related problems such as optimal control are much more difficult to solve than those of linear systems. In literature, many authors [5-9] have tried various methods to overcome the difficulties of solving bilinear systems. In this paper, the focus will be on obtaining the optimal state solution of a wave equation governed by a bilinear system using CAS wavelets taking advantage of some needed properties of this type of wavelets $[10,11]$. Compared to conventual method such as Fourier series or finite elements, CAS wavelets with their local properties enable arbitrary functions (even with discontinuity) to be approximated more efficient. To demonstrate the effectiveness of the proposed approach, numerical results will show confirm that the proposed method significantly minimizes the energy of the system.

\section{Optimal Control Problem Setting}

Let $\Omega_{x}$ be an open, bounded, and simply connected subset of $n$-dimensional Euclidean space $\mathbb{R}^{n}$. Let $\Omega_{t}$ denote a given time interval $\left(0, t_{f}\right)$ with finite terminal time $t_{f}$. Consider the wave equation, defined on $Q=\Omega_{x} \times \Omega_{t}$ :

$$
u_{t t}=a(t) \Delta u,
$$

where $\Delta$ is the Laplacian operator, and $u(x, t)$ is the disturbance of position $x$ and time $t$. The wave speed $\sqrt{a(t)}$ is assumed to be a function of time. For simplicity, let $u$ satisfy the boundary and initial conditions:

$$
\begin{gathered}
u(x, t)=0, \quad \Delta u=0, \quad \forall x \in \partial \Omega_{x}, \\
u(x, 0)=w_{0}(x), \quad u_{t}(x, 0)=w_{1}(x), \quad x \in \Omega_{x},
\end{gathered}
$$

where

$$
w_{0}(x) \in H^{2}\left(\Omega_{x}\right)=\left\{h(x): \frac{\partial^{i} h}{\partial x^{i}} \in L^{2}\left(\Omega_{x}\right), i=1,2\right\}
$$

and $w_{1}(x) \in L^{2}\left(\Omega_{x}\right)$. 
Let the admissible control set be

$$
A_{\mathrm{ad}}=\left\{a(t): a(t) \in L^{2}\left(\Omega_{t}\right)\right\}
$$

Associated with the wave equation (2.1) is the modified energy $J[a(t)]$ at terminal time $t_{f}$ :

$$
J[a(t)]=\mu_{1} \int_{\Omega_{x}} u^{2}\left(x, t_{f}\right) d x+\mu_{2} \int_{\Omega_{x}} u_{t}^{2}\left(x, t_{f}\right) d x+\mu_{3} \int_{\Omega_{t}} a^{2}(t) d t
$$

where $\mu_{1}, \mu_{2}$, and $\mu_{3}$ are weighing constants satisfying the condition $\mu_{1}+\mu_{2}>0$ and $\mu_{3}>0$. The last term on the right-hand side of (2.5) is a penalty term on control energy.

The optimal control problem is stated as follows: determine the optimal control function $a^{*}(t) \in A_{\text {ad }}$ such that

$$
J\left[a^{*}(t)\right]=\min _{a(t) \in A_{\mathrm{ad}}} J[a(t)]
$$

subject to (2.1) and (2.2).

\section{Control Problem in Modal Space}

We pose the problem at hand as a control problem for an finite system of ordinary differential equations by using modal space expansion. Let

$$
u(x, t)=\sum_{n=1}^{N} z_{n}(t) \varphi_{n}(x)
$$

where $\varphi_{n}(x)$ are normalized eigenfunctions associated with eigenvalues $w_{n}^{2}$. This implies that $\varphi_{n}(x)$ satisfies the eignvalue-problem

$$
\begin{gathered}
\Delta \varphi_{n}(x)+w_{n}^{2} \varphi_{n}(x)=0, \quad x \in \Omega_{x}, \\
\varphi_{n}(x)=0, \quad x \in \partial \Omega_{x}, \\
\Delta \varphi_{n}(x)=0, \quad x \in \partial \Omega_{x} .
\end{gathered}
$$

It can be shown that the set $\varphi_{n}(x)$ forms an orthonormal set, and hence $z_{n}(t)$ satisfies

$$
\frac{d^{2}}{d t^{2}} z_{n}(t)+a(t) w_{n}^{2} z_{n}(t)=0, \quad n=1,2, \ldots, N
$$

with initial conditions

$$
z_{n}(0)=z_{0 n}, \quad \frac{d}{d t} z_{n}(0)=z_{1 n}, \quad n=1, \ldots, N
$$


In view of the expansion (3.1), the performance index becomes

$$
J_{N}[a(t)]=\sum_{n=1}^{N}\left[\mu_{1} z_{n}^{2}\left(t_{f}\right)+\mu_{2}\left(\frac{d}{d t} z_{n}\left(t_{f}\right)\right)^{2}\right]+\mu_{3} \int_{0}^{t_{f}} a^{2}(t) d t
$$

The optimal control problem (2.6) is now modified as follows: determine $a^{*}(t) \in A_{\text {ad }}$ such that

$$
J_{N}\left[a^{*}(t)\right]=\min _{a(t) \in A_{\mathrm{ad}}} J_{N}[a(t)]
$$

subject to (3.3) and (3.4).

\section{Properties of the CAS Wavelets}

\subsection{CAS Wavelets}

Wavelets have been used by many scientists and engineers to solve several problems in areas such as signal and image processing, control problems, and stochastic problems. Wavelets are mathematical functions that are constructed using dilation and translation of a single function called the mother wavelet denoted by $\psi(t)$ and must satisfy certain requirements. If the dilation parameter is $a$ and translation parameter is $b$, then we have the following family of wavelets:

$$
\psi_{a, b}(t)=|a|^{-1 / 2} \psi\left(\frac{t-b}{a}\right), \quad \text { with } a, b \in R, a \neq 0
$$

Restricting $a$ and $b$ to discrete values, such as $a=a_{0}^{-k}, b=n b_{0} a_{0}^{-k}, a_{0}>1, b_{0}>0$ and $n$ and $k$ are positive integers, gives

$$
\psi_{k, n}(t)=\left|a_{0}\right|^{k / 2} \psi\left(a_{0}^{k} t-n b_{0}\right)
$$

where $\psi_{k, n}(t)$ form a basis for $L^{2}(R)$. If $a_{0}=2$ and $b_{0}=1$, then it is clear that the set $\left\{\psi_{k, n}(t)\right\}$ forms an orthonormal basis for $L^{2}(R)$.

The CAS wavelets employed in this paper are defined as

$$
\psi_{n, m}(t)= \begin{cases}2^{k / 2} \mathrm{CAS}_{m}\left(2^{k} t-n\right), & \text { if } \frac{n}{2^{k}} \leq t<\frac{n+1}{2^{k}} \\ 0, & \text { otherwise }\end{cases}
$$

where

$$
\mathrm{CAS}_{m}(t)=\cos (2 m \pi t)+\sin (2 m \pi t) .
$$


The set of CAS wavelets forms an orthonormal basis for $L^{2}([0,1])$. This implies that any function $f(t)$ defined over $[0,1]$ can be expanded as

$$
\begin{aligned}
f(t) & =\sum_{n=0}^{\infty} \sum_{m \in Z} d_{n, m} \psi_{n, m}(t) \\
& \simeq \sum_{n=0}^{2^{k}-1} \sum_{m=-M}^{M} d_{n, m} \psi_{n, m}(t)=D^{T} \Psi(t),
\end{aligned}
$$

where

$$
d_{n, m}=\left(f(t), \psi_{n, m}(t)\right)=\int_{0}^{1} f(t) \psi_{n, m}(t) d t
$$

and $D$ and $\Psi(t)$ are $2^{k}(2 M+1) \times 1$ vectors given by

$$
\begin{aligned}
D & =\left[d_{0,-M}, d_{0,(-M+1)}, \ldots, d_{0, M}, d_{1,-M}, \ldots, d_{1, M}, \ldots, d_{\left(2^{k}-1\right),-M}, \ldots, d_{\left(2^{k}-1\right), M}\right]^{T}, \\
\Psi(t) & =\left[\Psi_{0,-M}, \Psi_{0,(-M+1)}, \ldots, \Psi_{0, M}, \Psi_{1,-M}, \ldots, \Psi_{1, M}, \ldots, \Psi_{\left(2^{k}-1\right),-M}, \ldots, \Psi_{\left(2^{k}-1\right), M}^{T}\right] .
\end{aligned}
$$

\subsection{Operational Matrices of Integration}

The integration of the function $\Psi(t)$ in (4.5) is given by

$$
\int_{0}^{t} \Psi(s) d s=P \Psi(t)
$$

where $P$ is an $2^{k}(2 M+1) \times 2^{k}(2 M+1)$ matrix, called the operational matrix, and is given by [12]

$$
P=\frac{1}{2^{k+1}}\left[\begin{array}{ccccc}
S & F & F & \cdots & F \\
O & S & F & \cdots & F \\
\vdots & O & \ddots & \ddots & \vdots \\
& & & & F \\
O & O & \cdots & O & S
\end{array}\right]
$$


in which $O$ is a zero matrix and $F$ and $S$ are $(2 M+1) \times(2 M+1)$ matrices given by

$$
\begin{aligned}
& \quad F=\left[\begin{array}{cccccc}
0 & \cdots & \cdots & \cdots & 0 \\
\vdots & \ddots & \vdots & \ddots & \\
0 & \cdots & 2 & \cdots & 0 \\
\vdots & \vdots & \vdots & \ddots & \ddots \\
0 & \cdots & \cdots & \cdots & 0
\end{array}\right], \\
& S=\left[\begin{array}{cccccccccc}
0 & 0 & \cdots & 0 & -\frac{1}{M \pi} & 0 & \cdots & 0 & \frac{1}{M \pi} \\
0 & 0 & \cdots & 0 & -\frac{1}{(M-1) \pi} & 0 & \cdots & \frac{1}{(M-1) \pi} & 0 \\
\cdots & \cdots & \cdots & \cdots & \cdots & \cdots & \cdots & \cdots & \cdots \\
0 & 0 & \cdots & 0 & -\frac{1}{\pi} & \frac{1}{\pi} & \cdots & 0 & 0 \\
\frac{1}{\pi} & \frac{1}{\pi} & \cdots & \frac{1}{\pi} & 1 & \frac{1}{\pi} & \cdots & \frac{1}{\pi} & \frac{1}{\pi} \\
0 & 0 & \cdots & \frac{1}{\pi} & \frac{1}{\pi} & 0 & \cdots & 0 & 0 \\
\cdots & \cdots & \cdots & \cdots & \cdots & \cdots & \cdots & \cdots & \cdots \\
0 & \frac{1}{(M-1) \pi} & \cdots & 0 & \frac{1}{(M-1) \pi} & 0 & \cdots & 0 & 0 \\
\frac{1}{M \pi} & 0 & \cdots & 0 & \frac{1}{M \pi} & 0 & \cdots & 0 & 0
\end{array}\right] .
\end{aligned}
$$

The integration of the product of two CAS function vectors is given by

$$
\int_{0}^{1} \Psi(t) \Psi(t)^{T} d t=I
$$

The product operational matrix of the CAS wavelet is given by

$$
\Psi(t) \Psi(t)^{T} C \simeq \widetilde{C} \Psi(t)
$$

where the matrix $C$ is given in (4.7) and $\tilde{C}$ is an $2^{k}(2 M+1) \times 2^{k}(2 M+1)$ given by [11]

$$
\widetilde{C}=\left[\begin{array}{cc}
\widetilde{C_{1}} & 0 \\
0 & \widetilde{C_{2}}
\end{array}\right],
$$


where $\widetilde{C_{i}}, i=1,2$ are $(2 M+1) \times(2 M+1)$ given by

$$
\widetilde{C_{i}}=\left[\begin{array}{ccc}
c_{i, 0} & c_{i,-1} & 0 \\
c_{i,-1} & c_{i, 0} & c_{i, 1} \\
0 & c_{i, 1} & c_{i, 0}
\end{array}\right] \quad \text { for } i=1,2
$$

\section{CAS-Wavelets-Based Approach}

To redefine the wavelet functions over the interval $\left[0, t_{f}\right]$, we let $t=t_{f} \tau$. Then (3.3), (3.4), and (3.5), respectively, become

$$
\begin{gathered}
\frac{d^{2}}{d \tau^{2}} z_{i}(\tau)+w_{i}^{2} t_{f}^{2} a(\tau) z_{i}(\tau)=0, \quad \text { for } 0<\tau<1, i=1,2, \ldots, N, \\
z_{i}(0)=z_{i 0}, \quad \frac{d}{d \tau} z_{i}(0)=t_{f} z_{i 1}, \quad i=1, \ldots, N, \\
J_{N}(a(\tau))=\sum_{i=1}^{N}\left[\mu_{1} z_{i}^{2}\left(t_{f}\right)+t_{f}^{2} \mu_{2} \frac{d}{d \tau} z_{i}^{2}\left(t_{f}\right)\right]+t_{f} \mu_{3} \int_{0}^{1} a^{2}(\tau) d \tau .
\end{gathered}
$$

Using the expansion in (4.5) gives

$$
\begin{aligned}
\frac{d^{2}}{d \tau^{2}} z_{i}(\tau) & =\vec{B}_{i}^{T} \vec{\Psi}(\tau)=\overrightarrow{\Psi^{T}}(\tau) \vec{B}_{i} \\
a(\tau) & =\vec{C}^{T} \vec{\Psi}(\tau)=\vec{\Psi}(\tau) \vec{C} \\
z_{i}(0) & =\vec{\Phi}_{i}^{T} \vec{\Psi}(\tau)=\overrightarrow{\Psi T}(\tau) \vec{\Phi}_{i} \\
\frac{d}{d \tau} z_{i}(0) & =\vec{\digamma}_{i}^{T} \vec{\Psi}(\tau)=\vec{\Psi}(\tau) \vec{\digamma}_{i}
\end{aligned}
$$

where $\vec{B}_{i}, \vec{C}, \vec{\Phi}_{i}, \vec{F}_{i}$, and $\vec{\Psi}(\tau)$ are $2^{k}(2 M+1) \times 1$ vectors defined as in (4.7). Furthermore,

$$
\begin{aligned}
\frac{d}{d \tau} z_{i}(\tau) & =\int_{0}^{\tau} \frac{d^{2}}{d s^{2}} z_{i}(s) d s+\frac{d}{d \tau} z_{i}(0) \\
& =\int_{0}^{\tau} \vec{B}_{i}^{T} \vec{\Psi}(s) d s+\vec{\digamma}_{i}^{T} \vec{\Psi}(\tau) \\
& \left.=\vec{B}_{i}^{T} P \Psi \overrightarrow{(\tau}\right)+\vec{\digamma}_{i}^{T} \vec{\Psi}(\tau)
\end{aligned}
$$




$$
\begin{aligned}
z_{i}(\tau) & =\int_{0}^{\tau} \frac{d}{d \tau} z_{i}(s) d s+z_{i}(0) \\
& =\int_{0}^{\tau}\left(\vec{B}_{i}^{T} P \Psi \vec{P}(s)+\vec{\digamma}_{i}^{T} \vec{\Psi}(s)\right) d s+\vec{\Phi}_{i}^{T} \vec{\Psi}(\tau) \\
& \left.=\vec{B}_{i}^{T} P^{2} \Psi \overrightarrow{(\tau}\right)+\vec{\digamma}_{i}^{T} \overrightarrow{P \Psi}(\tau)+\vec{\Phi}_{i}^{T} \vec{\Psi}(\tau) \\
& \left.=\Psi^{T}(\tau) \overrightarrow{\left(P^{2}\right.}\right)^{T} \vec{B}_{i}+\vec{\Psi}^{T}(\tau) P^{T} \vec{\digamma}_{i}+\vec{\Psi}^{T}(\tau) \vec{\Phi}_{i} .
\end{aligned}
$$

Substituting (5.5) in (5.1) yields

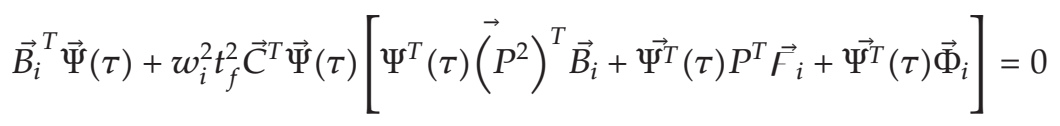

and hence

$$
\begin{aligned}
& \vec{B}_{i}^{T} \vec{\Psi}(\tau)+w_{i}^{2} t_{f}^{2} \vec{C}^{T} \vec{\Psi}(\tau) \Psi^{T}(\tau)\left(P^{2}\right)^{T} \vec{B}_{i} \\
& \quad+w_{i}^{2} t_{f}^{2} \vec{C}^{T} \vec{\Psi}(\tau) \overrightarrow{\Psi^{T}}(\tau) P^{T} \vec{\digamma}_{i}+w_{i}^{2} t_{f}^{2} \vec{C}^{T} \vec{\Psi}(\tau) \Psi^{T}(\tau) \vec{\Phi}_{i}=0 .
\end{aligned}
$$

Using (4.12) leads to

$$
\vec{B}_{i}^{T} \vec{\Psi}(\tau)+w_{i}^{2} t_{f}^{2} \overrightarrow{\Psi^{T}}(\tau) \tilde{C}^{T}\left(P^{2}\right)^{T} \vec{B}_{i}+w_{i}^{2} t_{f}^{2} \overrightarrow{\Psi^{T}}(\tau) \tilde{C}^{T} P^{T} \vec{\digamma}_{i}+w_{i}^{2} t_{f}^{2} \vec{\Psi}^{T}(\tau) \tilde{C}^{T} \vec{\Phi}_{i}=0
$$

Multiplying (5.8) by $\vec{\Psi}(\tau)$, integrating, and using (4.11) give

$$
\vec{B}_{i}+w_{i}^{2} t_{f}^{2} \tilde{C}^{T}\left(P^{2}\right)^{T} \vec{B}_{i}+w_{i}^{2} t_{f}^{2} \tilde{C}^{T} P^{T} \vec{\digamma}_{i}+w_{i}^{2} t_{f}^{2} \tilde{C}^{T} \vec{\Phi}_{i}=0
$$

or

$$
\vec{B}_{i}=-G^{-1}\left(w_{i}^{2} t_{f}^{2} \tilde{C}^{T} P^{T} \vec{\digamma}_{i}+w_{i}^{2} t_{f}^{2} \tilde{C}^{T} \vec{\Phi}_{i}\right)
$$

provided that

$$
G=\left(I+w_{i}^{2} t_{f}^{2} \tilde{C}^{T}\left(P^{2}\right)^{T}\right)^{-1}
$$

exists. Substituting equations (5.4) and (5.5) into equation (3.5) convert the performance index $J_{N}[a(t)]$ into a function of $\widetilde{C}$ and hence to optimize $J_{N}[a(t)]$, we solve

$$
\frac{\partial J_{N}}{\partial c_{i}}=0
$$


Table 1: Comparison between uncontrolled and controlled performance indices.

\begin{tabular}{lc}
\hline Controller $a(t)$ & $J_{1}[a(t)]$ \\
\hline$a^{*}(t)$ & 0.02451 \\
$t$ & 0.50858 \\
$t^{2}$ & 0.36656 \\
$\sin t$ & 0.44015 \\
$\cos t$ & 3.62556 \\
\hline
\end{tabular}

\section{Numerical Example}

Consider the wave equation

$$
\frac{d^{2}}{d \tau^{2}} z(\tau)+w^{2} t_{f}^{2} a(\tau) z(\tau)=0, \quad \text { for } 0<\tau<1
$$

with initial conditions

$$
z(0)=1, \quad \frac{d}{d \tau} z(0)=0
$$

For the sake of illustration, the following parameters were assumed:

$$
\begin{gathered}
w_{1}=\pi, \quad t_{f}=1, \quad \mu_{1}=\mu_{2}=\mu_{3}=1, \quad \Omega_{x}=(0,1), \\
M=1, \quad k=1 \text { (6 wavelet expansions). }
\end{gathered}
$$

The performance index was computed for the optimal control $a^{*}(t)$ and compared with the performance index for the controllers $a(t)=t, a(t)=t^{2}, a(t)=\sin t$, and $a(t)=\cos t$. The results are summarized in Table 1.

It is observed that the proposed control is effective in significantly reducing the performance index of the problem.

\section{Conclusion}

A control for a wave equation where the control is a time dependent coefficient is considered. A modal space technique simplifies the optimal control of a distributed parameter system into the optimal control of a bilinear time-invariant lumped-parameter system. A Galerkin CAS wavelet-based method was developed to solve this bilinear optimal control problem. The main aspect of the proposed approach resides in converting the optimization problem into a mathematical programming problem where the necessary conditions of optimality are derived as a system of algebraic equations. A test example, which includes a variable coefficient and one-dimensional hyperbolic equation, demonstrates the capability of the proposed Galerkin-Wavelet approach for solving optimal control problems governed by bilinear systems. Moreover, the numerical simulations show that the optimal control procedure led to a substantial damping in the bilinear system energy. 
This method may be extended to treat a more general setting where the coefficients are $x$ and $t$ dependent. That is, the wave speed function is a controllable function of the form $a(x, t)[13]$.

\section{References}

[1] V. Berdichersky, V. Jikov, and G. Papanicolcou, Eds., Homogenization, vol. 50 of Series on Advances in Mathematics for Applied Sciences, World Scientific Publishing, River Edge, NJ, USA, 1999.

[2] K. A. Lurie, "Control in the coefficients of linear hyperbolic equations via spacio-temporal components," in Homogenization, vol. 50 of Series on Advances in Mathematics for Applied Sciences, pp. 285-315, World Scientific, River Edge, NJ, USA, 1999.

[3] K. A. Lurie, "Some new advances in the theory of dynamic materials," Journal of Elasticity, vol. 72, no. 1-3, pp. 229-239, 2003.

[4] S. Hurlebaus and L. Gaul, "Smart structure dynamics," Mechanical Systems and Signal Processing, vol. 20, no. 2, pp. 255-281, 2006.

[5] A. Chambolle and F. Santosa, "Control of the wave equation by time-dependent coefficient," ESAIM: Control, Optimisation and Calculus of Variations, vol. 8, pp. 375-392, 2002.

[6] H. R. Joshi, "Optimal control of the convective velocity coefficient in a parabolic problem," Nonlinear Analysis: Theory, Methods \& Applications, vol. 63, no. 5-7, pp. 1383-1390, 2005.

[7] S. Lenhart, M. Liang, and V. Protopopescu, "Identification problem for a wave equation via optimal control," in Control of Distributed Parameter and Stochastic Systems, vol. 141 of IFIP Conference Proceedings, pp. 79-84, Kluwer Academic Publishers, Boston, Mass, USA, 1999.

[8] F. Maestre, A. Münch, and P. Pedregal, "A spatio-temporal design problem for a damped wave equation," SIAM Journal on Applied Mathematics, vol. 68, no. 1, pp. 109-132, 2007.

[9] M. Tatari and M. Dehghan, "Identifying a control function in parabolic partial differential equations from over specified boundary data," Computers $\mathcal{E}$ Mathematics with Applications, vol. 53, no. 12, pp. 1933-1942, 2007.

[10] M. Razzaghi and S. Yousefi, "Sine-cosine wavelets operational matrix of integration and its applications in the calculus of variations," International Journal of Systems Science, vol. 33, no. 10, pp. 805-810, 2002.

[11] S. Yousefi and A. Banifatemi, "Numerical solution of Fredholm integral equations by using CAS wavelets," Applied Mathematics and Computation, vol. 183, no. 1, pp. 458-463, 2006.

[12] H. Danfu and S. Xufeng, "Numerical solution of integro-differential equations by using CAS wavelet operational matrix of integration," Applied Mathematics and Computation, vol. 194, no. 2, pp. 460-466, 2007.

[13] M. Liang, "Bilinear optimal control for a wave equation," Mathematical Models $\mathcal{E}$ Methods in Applied Sciences, vol. 9, no. 1, pp. 45-68, 1999. 


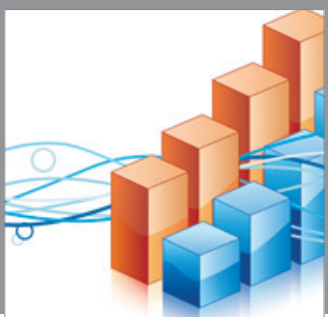

Advances in

Operations Research

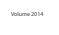

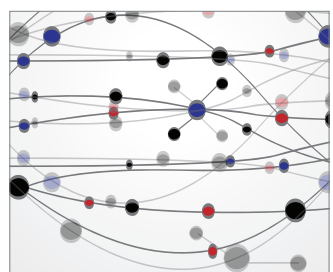

\section{The Scientific} World Journal


International Journal of

Mathematics and

Mathematical

Sciences
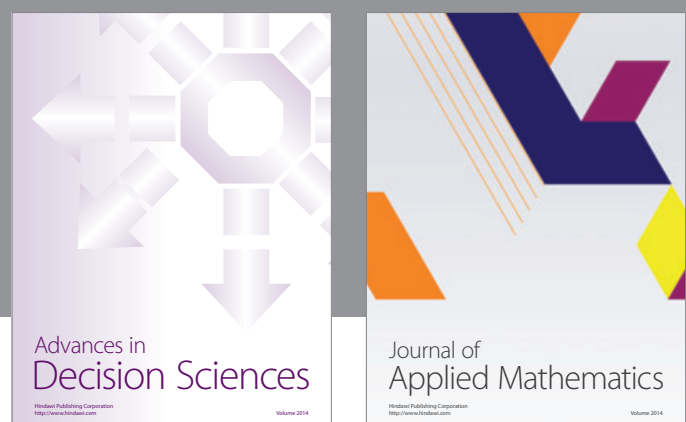

Journal of

Applied Mathematics
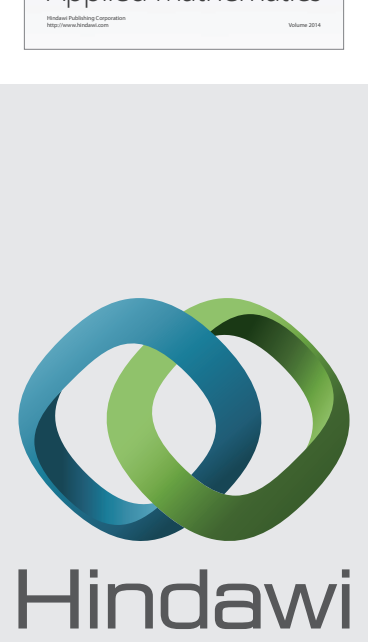

Submit your manuscripts at http://www.hindawi.com
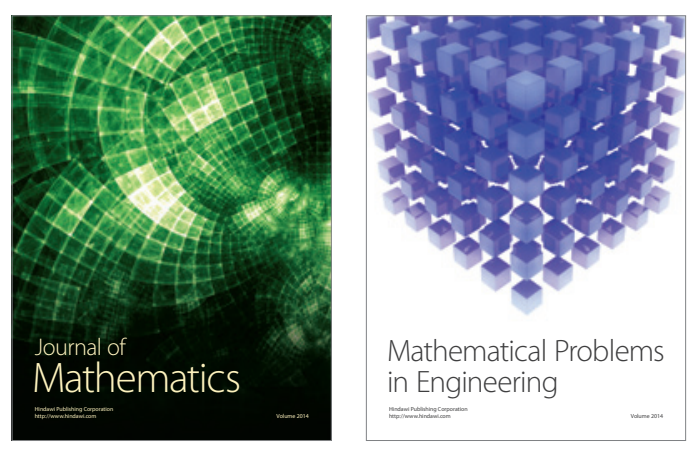

Mathematical Problems in Engineering
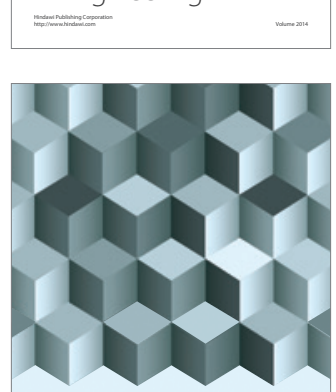

Journal of

Function Spaces
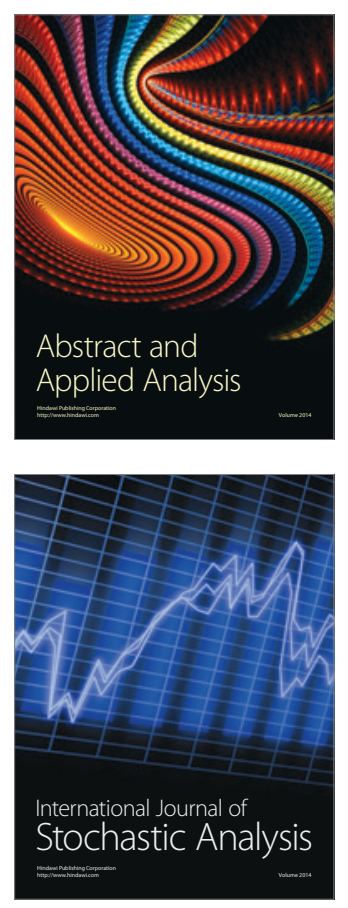

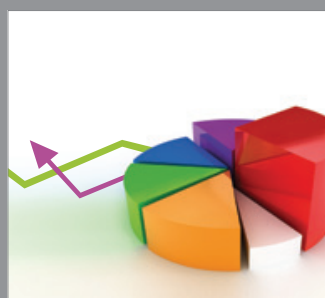

ournal of

Probability and Statistics

Promensencen
